\title{
ORIGINAL
}

\section{Traumatismo craneoencefálico pediátrico grave (II): factores relacionados con la morbilidad y mortalidad}

\author{
J.M. López Álvarez ${ }^{a, *}$, M.E. Valerón Lemaur ${ }^{a}$, O. Pérez Quevedo ${ }^{a}$, J.M. Limiñana Cañal ${ }^{b}$, \\ A. Jiménez Bravo de Laguna ${ }^{a}$, E. Consuegra Llapurt ${ }^{a}, A$. Morón Saén de Casas ${ }^{a}$ y \\ R. González Jorge ${ }^{a}$
}

a Unidad de Medicina Intensiva Pediátrica, Complejo Hospitalario Universitario Insular Materno-Infantil de Canarias, Las Palmas de Gran Canaria, Las Palmas, España

b Departamento de Bioestadística, Facultad de Medicina, Universidad de Las Palmas de Gran Canaria, Las Palmas, España

Recibido el 21 de septiembre de 2010; aceptado el 1 de febrero de 2011

Disponible en Internet el 14 de abril de 2011

\section{PALABRAS CLAVE \\ Traumatismo craneoencefálico pediátrico; Factores pronósticos; Morbilidad; Mortalidad}

\begin{abstract}
Resumen
Objetivo: Describir los factores asociados a la morbilidad y mortalidad de los pacientes pediátricos con traumatismo craneoencefálico grave (TCEG).

Material y método: Revisión de los pacientes ingresados en una unidad de medicina intensiva pediátrica (UMIP) con TCEG en el periodo comprendido entre julio de 1983 y diciembre de 2009. Resultados: De los 389 pacientes con TCE ingresados en nuestra unidad durante el periodo de estudio, presentaron TCEG 174 (45\%). La edad media de este grupo fue de $67 \pm 9$ meses, con una puntuación media en la escala de Glasgow (GCS) de 5,5 $+1,8$ y una puntuación PRISM media de $10,6 \pm 6,7$. El $39 \%$ de los pacientes presentaron lesión encefálica difusa (LED) grave en la TAC. Un $79 \%$ de los pacientes en los que se monitorizó la presión intracraneal (PIC) presentaron hipertensión intracraneal (HIC). Estos pacientes tuvieron una mayor incidencia de secuelas graves que aquellos que no desarrollaron HIC $(66,7$ vs $23,1 \%$; $p=0,01)$.

Las secuelas de relevancia clínica se encontraron en 59 pacientes $(34 \%)$, y fueron graves en el $64 \%$ de los mismos.

La mortalidad de los pacientes con TCEG fue de un $25 \%$ y se asoció de forma significativa a una menor puntuación del GCS, a la existencia de hiperglucemia o HIC, a la presencia de midriasis o shock y a la necesidad de ventilación mecánica. La mortalidad de la LED grave fue significativamente más elevada que la LED leve-moderada $(87,5$ vs $7,2 \%$; $p<0,001)$ y que la lesión focal $(87,5$ vs $36,1 \% ; p<0,001)$. Los factores responsables de la mortalidad de forma independiente en los pacientes pediátricos con TCEG fueron la existencia de midriasis (OR: 31,27), HIC (OR: 13,23) e hiperglucemia (OR: 3,10).
\end{abstract}

\footnotetext{
* Autor para correspondencia.

Correo electrónico: jmloal@hotmail.com (J.M. López Álvarez).
}

0210-5691/\$ - see front matter @ 2010 Elsevier España, S.L. y SEMICYUC. Todos los derechos reservados. doi:10.1016/j.medin.2011.02.006 


\section{KEYWORDS}

Severe head injury pediatric patients; Risk factors; Morbidity; Mortality
Conclusiones: a) Los TCEG en edad pediátrica asocian una alta morbilidad y mortalidad; b) la existencia de HIC se asoció al desarrollo de secuelas graves; c) los factores de riesgo de mortalidad de forma independiente fueron la existencia de midriasis, HIC e hiperglucemia. (c) 2010 Elsevier España, S.L. y SEMICYUC. Todos los derechos reservados.

\section{Severe pediatric head injuries (II): factors associated to morbidity-mortality}

\begin{abstract}
Objective: To describe the factors associated to morbidity-mortality in pediatric patients with severe head injury $(\mathrm{SHI})$.

Material and method: A review was made of the patients admitted to the Pediatric Intensive Care Unit (PICU) with SHI between July 1983 and December 2009.

Results: Of the 389 patients with head injuries, 174 (45\%) presented SHI. The mean age of these subjects was 67 (9) months, with a Glasgow Coma Score (GCS) of 5.5 (1.8) and a PRISM score of 10.6 (6.7). Thirty-nine percent of the patients showed diffuse encephalic injury (DEI) in the computed tomography (CT) study. Seventy-nine percent of the patients subjected to intracranial pressure monitoring (ICP) presented intracranial hypertension. These patients had a greater incidence of serious sequelae $(66.7$ vs. $23.1 \%$; $=0.01)$. Sequelae of clinical relevance were recorded in 59 patients (34\%), and proved serious in $64 \%$ of the cases. The mortality rate among the patients with $\mathrm{SHI}$ was $24.7 \%$, and mortality was significantly associated with a lower GCS score, hyperglycemia, intracranial hypertension and the presence of mydriasis or shock. The mortality rate associated to severe DEI was significantly higher than in the case of mild-moderate DEI (87.5 vs. $7.2 \%$; $p<0.001)$.

The independent mortality risk factors in the pediatric patients with $\mathrm{SHI}$ were found to be the presence of mydriasis (OR: 31.27), intracranial hypertension (OR: 13.23) and hyperglycemia (OR: 3.10).

Conclusions: a) SHI in pediatric patients was associated with high morbidity-mortality; $b$ ) intracranial hypertension was associated to the development of serious sequelae; c) independent mortality risk factors were the existence of mydriasis, intracranial hypertension and hyperglycemia.
\end{abstract}

(c) 2010 Elsevier España, S.L. and SEMICYUC. All rights reserved.

\section{Introducción}

En la población pediátrica el traumatismo craneoencefálico (TCE) es la principal causa de muerte traumática y además es el responsable de secuelas tan graves como el retraso mental, la epilepsia infantil y la incapacidad física ${ }^{1-3}$.

El manejo adecuado de los niños que sufren un traumatismo craneoencefálico grave (TCEG) es de vital importancia para minimizar las complicaciones del mismo.

La morbilidad y mortalidad del TCE viene determinada por las lesiones primarias que se producen en el momento del traumatismo (relacionadas con el tipo de traumatismo y localización) y por las lesiones secundarias (hipoxia, isquemia, edema, hipertensión intracraneal [HIC]) cuyo efecto aparece tardíamente y sobre las que podemos actuar de forma preventiva mediante una reanimación y estabilización adecuada $^{4-6}$.

Los avances en el conocimiento fisiopatológico de los eventos que se producen tras el TCE han permitido el desarrollo de nuevas medidas diagnósticas y terapéuticas. A pesar de ello su morbilidad y mortalidad continúa siendo alta $^{7,9}$. Por este motivo se insiste en la búsqueda de sistemas de monitorización de la dinámica cerebral y de predicción pronóstica $^{6,10,11}$.

El objetivo del presente estudio ha sido: analizar los factores que influyen en la morbilidad y mortalidad de los pacientes pediátricos con TCEG.

\section{Material y métodos}

\section{Estudio}

Retrospectivo (agosto de 1983-diciembre de 1998), prospectivo (enero de 1999-diciembre de 2009).

\section{Ámbito}

- Población de referencia: niños con edad comprendida entre un mes y 14 años, que han sufrido un TCE, con puntuación GCS menor o igual a 8 .

- Centro hospitalario: hospital de tercer nivel, centro de referencia provincial (Islas de Gran Canaria, Fuerteventura y Lanzarote) para una población infantil de 137.538 niños.

\section{Variables analizadas}

- Variables cuantitativas: edad, puntuación de la escala de Glasgow (GCS), puntuación del índice de riesgo mortalidad pediátrica (PRISM), tiempo de ventilación mecánica tiempo de estancia en UMI, Glagow Outcome Scale (GOS).

- Variables cualitativas: sexo, procedencia, mortalidad, causa del TCE, lesiones asociadas extracraneales, lesio- 
nes en la TAC craneal, secuelas observadas, existencia de hiperglucemia, anemia, shock o midriasis pupilar, necesidad de monitorización de la presión intracraneal (PIC) y saturación venosa yugular de oxígeno $\left(\mathrm{SyO}_{2}\right)$, presencia de HIC y necesidad de ventilación mecánica.

\section{Criterios utilizados}

- GCS: se utilizó el primer valor reflejado una vez estabilizado el paciente en la puntuación obtenida en la escala de Glasgow clásica ${ }^{12}$ o la modificada para pacientes pediátricos ${ }^{13}$. Esta última se diferencia de la primera únicamente en el apartado de respuesta verbal, que se puntúa: Balbuceos (5), llanto irritable (4), llanto al dolor (3), queja o suspiro al dolor (2), ninguna respuesta (1).

- TCEG: puntuación GCS menor o igual a 8.

- Puntuación PRISM: puntuación de riesgo de mortalidad pediátrica $^{14}$.

- Paciente politraumatizado ${ }^{15,16}$ : Lesión grave en al menos 2 regiones corporales (cráneo/cerebro, tórax, abdomen, sistema músculo-esquelético) o 3 fracturas mayores. Lesión grave es aquella que produce en: 1) cráneo: inconsciencia, o focalidad neurológica, rinorragia, otorragia o fractura facial; 2) tórax: fracturas costales, fractura de esternón, neumotórax, hemotórax, contusión pulmonar, rotura aórtica, taponamiento cardiaco o rotura diafragmática; 3) abdomen: laceración o contusión orgánica; y 4) sistema músculo-esquelético: fractura de cuerpo o arco vertebral, fractura de pelvis, fémur, tibia, húmero o amputación de miembros.

- Lesión en TAC: se utilizó la clasificación del Trauma Coma Data Bank $(T C D B)^{17,18}$. Se consideró lesión encefálica difusa (LED) grave a la LED III y a la LED IV.

- Shock: presión arterial sistólica menor de $55 \mathrm{mmHg}$ en menores de un año y menor de $65 \mathrm{mmHg}$ en mayores de un año, con repercusión orgánica y necesidad de administración de fluidoterapia (mayor o igual a $20 \mathrm{cc} / \mathrm{kg}$ ) y/o catecolaminas para su control ${ }^{14}$.

- Hiperglucemia: glucemia mayor a $200 \mathrm{mg} / \mathrm{dl}$.

- Ventilación mecánica: pacientes que precisaron ventilación con presión positiva intermitente en el momento del TCE o en relación con éste.

- Anemia: hemoglobina menor de 8 gr\% o necesidad de transfusión de concentrados de hematíes en cantidad mayor o igual a $10 \mathrm{cc} / \mathrm{kg}$.

- Monitorizacion de PIC: se realizó con distintos sistemas de presión y en distintas ubicaciones según el caso clínico o la disponibilidad técnica.

- HIC: PIC mayor de $20 \mathrm{mmHg}$ de forma mantenida a pesar del control de todos los factores intra o extracraneales que pudiesen influir en su medida.

- Midriasis: dilatación pupilar arreactiva uni o bilateral mayor a $4 \mathrm{~mm}$.

- $\mathrm{S}_{y} \mathrm{O}_{2}$ : monitorización tras canalización de la vena yugular interna de forma retrógrada con comprobación radiológica de localización en bulbo yugular. Las determinaciones se realizaron de forma intermitente.

- Diferencia arterioyugular de oxígeno $\left(\mathrm{Sa}-\mathrm{yO}_{2}\right)^{19}$ : diferencia entre $\mathrm{S}_{\mathrm{a}} \mathrm{O}_{2}$ y la $\mathrm{S}_{\mathrm{y}} \mathrm{O}_{2}$. Se consideraron 3 posibilidades: 1) hiperemia cerebral: $\mathrm{S}_{a-y} \mathrm{O}_{2}$ menor de $20 \%$; 2) isquemia cerebral: $\mathrm{S}_{\mathrm{a}-\mathrm{y}} \mathrm{O}_{2}$ mayor de $40 \%$; y 3) normal: $\mathrm{S}_{\mathrm{a}-\mathrm{y}} \mathrm{O}_{2}$ entre 20 y $40 \%$.
- Evolución clínica: al alta de UMIP se clasificó a los pacientes en 4 categorías adaptando la Glasgow Outcome Scale $\left.(\mathrm{GOS})^{20}: 1\right)$ fallecimiento; 2) estado vegetativo: incapacidad para actuar recíprocamente con el ambiente; o incapacidad severa: capaz de seguir órdenes, incapaz de vivir de forma independiente; 3) incapacidad moderada: capaz de vivir de independiente; y 4) recuperación buena: capaz de volver a su situación basal previa al TCE. Se consideraron secuelas graves la puntuación GOS de 2 .

- Grupos etarios: a) edad menor a 2 años; b) edad comprendida entre 2 años y 6 años; y $c$ ) edad superior a 6 años.

\section{Análisis estadístico}

Los datos obtenidos fueron procesados en el programa Statistical Package for Social Sciences (SPSS) versión 17.0. Utilizamos la prueba de la $\mathrm{t}$ de Student para comparar medias y la prueba de la ji al cuadrado o la prueba exacta de Fisher para comparar porcentajes. El nivel de significación de nuestro análisis fue de alfa: 0,05.

El análisis multivariable se realizó mediante regresión logística con el método stepwise, es decir, la selección de variables por pasos hacia delante, con comprobación de entrada basada en la significación del estadístico de puntuación y comprobación de eliminación, basada en la probabilidad del estadístico de razón de verosimilitud (estimaciones de máxima verosimilitud parcial). El nivel de significación fue de 0,05.

Se realizó así mismo un análisis de calibración mediante las pruebas de bondad de ajuste y medida del estadístico $\chi^{2}$ de Hosmer-Lemeshow, para valorar el ajuste del modelo.

\section{Resultados}

Un total de 389 pacientes pediátricos ingresaron con el diagnóstico de TCE. Un 45\%, es decir, 174 niños, presentaban un TCEG, predominando el sexo masculino (67\%). La edad media de este grupo de pacientes fue de 67,9 $\pm 41,6$ meses. La mayoría ingresaron en UMI procedentes del área de urgencias $(61,5 \%)$ seguidos por los trasladados de otros hospitales (38\%). La causa del TCEG fueron los accidentes de tráfico en un $56 \%$ de las ocasiones, seguidos de las precipitaciones (25\%), otro tipo de accidentes (17\%) y en último lugar las agresiones (2\%).

La estancia media en UMI fue de $9,1 \pm 13$ días, con una mediana de 5 días. La puntuación PRISM media fue de $10,77+6,7$ y el tiempo medio de ventilación mecánica $99,41+58 \mathrm{~h}$ con una mediana de $48 \mathrm{~h}$.

En la evolución clínica cabe destacar que 65 pacientes (37\%) presentaron situación de inestabilidad hemodinámica y shock y que la existencia de anemia e hiperglucemia se constató en 112 pacientes (66\%) y en 66 pacientes (38\%) respectivamente. El TCE fue puro en el $66 \%$ de los casos y asoció fractura craneal en el 55\% de los pacientes. El 92,5\% de la población estudiada precisó ventilación mecánica.

En la tabla 1 se exponen la variables de monitorización neurológica más importantes, destacando que la puntuación del GCS medio fue de $5,5 \pm 1,8$ presentando un $47 \%$ de los pacientes una puntuación de GCS menor o igual a 5. En 104 pacientes $(60 \%)$ con TCEG se monitorizó la PIC, objetiván- 
Tabla 1 Principales variables de monitorización neurológica en los pacientes pediátricos con TCEG.

\begin{tabular}{lr}
\hline Variable & $\mathrm{n}(\%)$ \\
\hline GCS & \\
$3-5$ & $82(47)$ \\
$6-8$ & $92(53)$ \\
Midriasis pupilar & $60(35)$ \\
Monitorizacion PIC & $104(60)$ \\
$\mathrm{HTC}$ & $82(79)$ \\
$\mathrm{S}_{a y} \mathrm{O}_{2}$ & $19(11)$ \\
\hline
\end{tabular}

dose HIC en un $79 \%$ de los mismos. En un $25 \%$ de los pacientes con $\mathrm{HIC}$ se monitorizó la $\mathrm{S}_{\mathrm{y}} \mathrm{O}_{2}$ predominando la situación de baja extracción cerebral de oxígeno (hiperemia cerebral) en un $70 \%$ respecto a la situación de alta extracción cerebral de oxígeno (isquemia cerebral) observada en un $20 \%$ de los pacientes.

En los hallazgos obtenidos en la TAC, en el 71,7\% éstos fueron lesiones encefálicas difusas (siendo graves el 55\%). En las lesiones focales $(28,3 \%)$ predominaron las lesiones focales evacuadas en el $75 \%$ de los casos.

En la figura 1 se presenta la distribución porcentual del GOS modificado de los pacientes al alta de UMIP destacando la alta morbilidad (GOS2 y GOS3: 34,3\%) y mortalidad (24,7\%) de la nuestra población.

Se analizaron una serie de variables clínicas clásicamente relacionadas con la morbilidad y mortalidad en el TCEP. Los pacientes que desarrollaron HIC presentaron una incidencia significativamente superior de secuelas graves $(\mathrm{GOS}=2)$, respecto a aquellos que no tuvieron $\operatorname{HIC}(66,7$ vs $23,1 \%$; $p=0,01)$.

No existieron diferencias significativas de mortalidad en función de la edad, ni de forma cuantitativa $(71,3 \pm 42,8$ meses vs $57,9 \pm 31,7$ meses; $p=0,06$ ), ni cualitativa en los 3 grupos etarios que se dividió la población (edad menor a 2 años: 26,7\%; edad comprendida entre 2 años y 6 años: $31,4 \%$; edad mayor a 6 años: $31,7 \%$; NS).

Los pacientes con menor puntuación del GCS presentaron globalmente una mortalidad más alta. El rango osciló entre un $5,6 \%$ para los pacientes con GCS de 8 y un $56,4 \%$ de mortalidad para los pacientes con GCS de 3 .

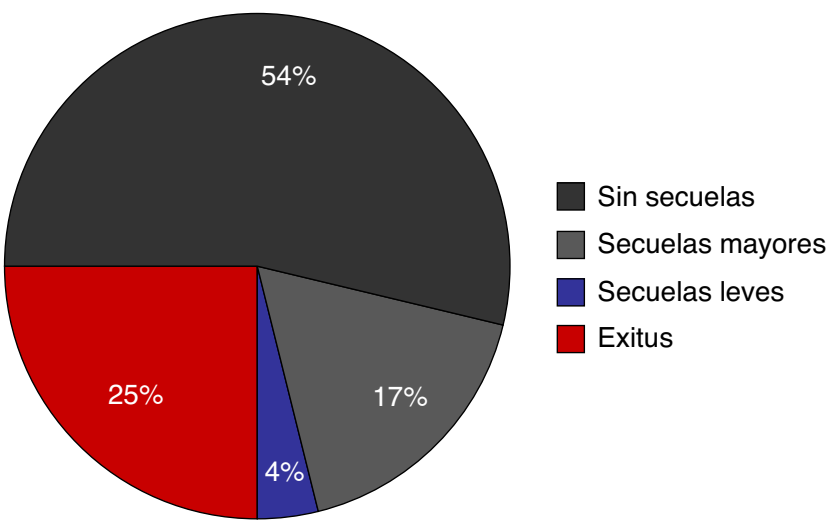

Figura 1 Evolución clínica de los pacientes pediátricos con TCEG según la escala GOS modificada.
Tabla 2 Factores asociados a la mortalidad en el TCEG pediátrico

\begin{tabular}{lccl}
\hline Variables & \multicolumn{2}{c}{ Exitus n (\%) } & $\mathrm{p}$ \\
\cline { 2 - 3 } & \multicolumn{1}{c}{ Si } & No & \\
\hline LED grave & $73(41,8)$ & $8(4,9)$ & $<0,001$ \\
Shock & $86(49,2)$ & $210,2)$ & $<0,001$ \\
Hiperglucemia & $74(42,4)$ & $25(14,2)$ & $<0,001$ \\
Anemia & $52(29,8)$ & $27(15,3)$ & $<0,05$ \\
Midriasis pupilar & $110(63,3)$ & $8(4,4)$ & $<0,001$ \\
HTC & $76(43,5)$ & $10(6 \%)$ & $<0,001$ \\
\hline
\end{tabular}

LED: Lesión encefálica difusa; HTC: Hipertensión intracraneal

Los pacientes que fallecieron tenían una mayor puntuación PRISM $(22,2+5,3$ vs $8,9+4,9 ; p<0,001)$ y una menor puntuación de GCS $(4,3 \pm 1,5$ vs $5,9 \pm 1,7 ; p<0,001)$. Así mismo en los pacientes que se constató anemia, shock, hiperglucemia, HIC o midriasis pupilar, la mortalidad fue de forma significativa más elevada (tabla 2 ). No encontramos diferencias en relación a la mortalidad en los pacientes con politraumatismo asociado, respecto a los que presentaron TCE puro (29 vs $23 \%$; NS), ni en los que presentaron fractura craneal, respecto a aquellos pacientes en los que ésta no se encontró (26 vs $23 \%$; NS).

En cuanto a la mortalidad asociada a cada una de las lesiones observadas en la TAC predominó la asociada a la LED IV con un $58 \%$ seguida de la LED III con un $29 \%$ y la LED $\mathrm{V}$ con un $28 \%$. La LED grave (III-IV) presentó una mortalidad significativamente superior a la LED I-II (41,8 vs 4,9; $\mathrm{p}<0,001)$.

La incidencia y mortalidad en distintos intervalos de tiempo durante la realización del estudio se presenta en la figura 2, destacando la tendencia a la disminución de la incidencia y mortalidad del TCEG pediátrico a lo largo del periodo de tiempo estudiado.

Se comparó la mortalidad en 2 periodos de tiempo (primeros 15 años de estudio respecto a la última década) y aunque existió una menor mortalidad en el segundo periodo estas diferencias no fueron significativas ( 25,5 vs $15,1 \%$; NS).

Se realizó un análisis multivariable para determinar los factores responsables de la mortalidad de forma independiente en los pacientes pediátricos con TCEG. Estos fueron la presencia de midriasis, HIC e hiperglucemia. En la tabla 3 se presentan estos factores junto al riesgo asociado a la mortalidad de cada uno de ellos.

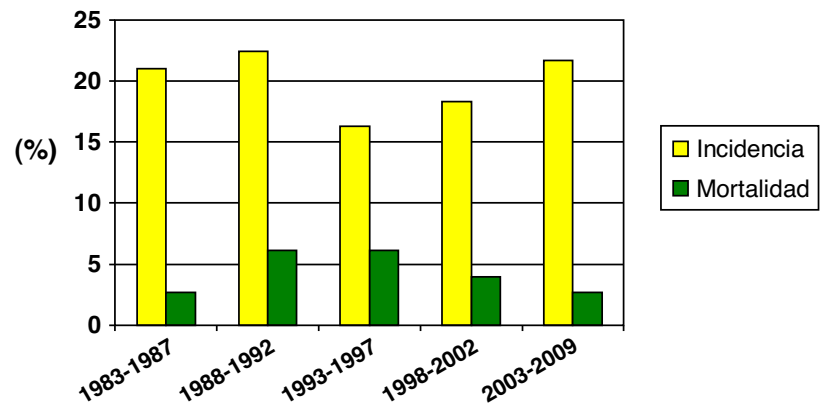

Figura 2 Distribución porcentual de la incidencia y mortalidad del TCEG pediátrico en los distintos periodos del estudio. 
Tabla 3 Factores independientes de mortalidad en el TCEG pediátrico

\begin{tabular}{lrrr}
\hline Variable & \multicolumn{1}{l}{ OR } & \multicolumn{1}{c}{ IC al 95\% } & \multicolumn{1}{c}{$\mathrm{p}$} \\
\hline Midriasis & 31,27 & {$[9,50-102,86]$} & 0,000 \\
HTC & 13,23 & {$[3,26-53,68]$} & 0,000 \\
Hiperglucemia & 3,10 & {$[1,01-9,48]$} & 0,048 \\
\hline
\end{tabular}

OR: Odds ratio; IC al 95\%: Intervalo de confianza al 95\%

Por último, se validó el buen ajuste del modelo mediante un análisis de calibración (Bondad de ajuste y estadístico $\chi^{2}$ de Hosmer-Lemeshow), obteniéndose un valor de $\chi^{2}$ de 2,31 y un valor de $\mathrm{p}$ de 0,80 .

\section{Discusión}

El estudio presentado recoge 174 TCEG pediátricos de un total de 389 niños con TCE (los últimos 10 años de forma prospectiva). Al intentar comparar nuestros resultados con los de otros autores ${ }^{7,8,21-25}$, las series no suelen ser homogéneas en aspectos como: a) la edad de los pacientes incluidos; b) la gravedad de los TCE; o c) la calidad de la asistencia pre o intrahospitalaria (medios de transporte, inmediatez de la reanimación, medios diagnósticos o terapéuticos, etc.).

Un primer aspecto que cabe destacar es la alta morbilidad de la población de estudio. Un tercio de la misma presentó secuelas de relevancia, siendo graves (coma vigil o secuelas incapacitantes) en un $22 \%$ de los niños analizados. A pesar de que la valoración se realizó en función de la puntuación GOS modificada (es decir al alta de UMIP y no a los 6 meses del TCE), este hecho nos obliga a realizar un máximo esfuerzo en prevenir la lesión secundaria asociada a todo TCE.

Entre los factores clásicamente asociados a la morbilidad del TCE pediátrico $9,18,26-29$, en nuestra serie sólo existió asociación significativa en los pacientes que desarrollaron HIC. Este dato es de vital importancia, porque la PIC se monitorizó en un $60 \%$ de los pacientes ingresados con TCEG y en ellos en el 79\% de los casos existió HIC. Esto nos debe obligar a la monitorización de la PIC en todo niño con TCEG.

La mortalidad de nuestra población es de un $25 \%$, porcentaje similar a otras series publicadas ${ }^{7,8,30}$. Sin embargo, la incidencia de TCEG en nuestro trabajo fue de un $45 \%$ destacando además que sólo se incluyeron a pacientes con edad comprendida entre un mes y 14 años. En el estudio de Ward et $\mathrm{al}^{31}$, se incluyen 201 pacientes con TCEG y la mortalidad tras 12 meses de seguimiento asciende a un $24 \%$, mientras que la reflejada por Feickert et $\mathrm{al}^{25}$ es del $22 \%$.

Entre las variables neurológicas, la puntuación GCS se asoció a una mayor mortalidad $(5,9 \pm 1,7$ vs $4,2 \pm 1,5$; $\mathrm{p}<0,001)$, destacando que el $100 \%$ de los pacientes que fallecieron presentaban un GCS menor o igual a 8 . En nuestra serie un $50 \%$ de los pacientes presentaron un GCS menor o igual a 5, asociando este grupo una mortalidad significativamente mayor que el grupo con GCS comprendido entre 6 y 8 (39 vs $12 \%$; $p<0,001)$.

Igualmente, la existencia de midriasis o LED grave en la TAC, indicativas de HIC o cuanto menos de edema cerebral difuso, se asoció a una mayor mortalidad. Fernández et $a^{23,34}$ demuestran esta asociación en el TCE pediátrico y aunque analizaron tanto la primera TAC como la segunda realizada al paciente, sólo objetivaron asociación lineal entre la TAC inicial y la evolución clínica.

Entre las variables sistémicas $9,35-37,39,40$ la existencia de hiperglucemia, shock o anemia se asociaron a una mayor mortalidad. Así, Mansfield et $\mathrm{al}^{38}$ refleja un aumento de la mortalidad del $150 \%$ en pacientes con TCEG y shock, lo que supone un ascenso de la mortalidad del 22 al $66 \%$ en pacientes pediátricos con TCEG. En esta misma dirección cabe destacar el reciente estudio de White et $\mathrm{al}^{9}$ en el que se concluye que niveles de presión arterial elevados se asociaron a una mayor supervivencia.

El mejor pronóstico del TCE pediátrico en relación a los pacientes adultos, se constata en múltiples estudios ${ }^{33,38,41}$ siendo mayor la mortalidad en las edades más bajas en la población pediátrica $38,42,43$. En este trabajo la mortalidad mayor la presentaron los pacientes menores de 6 años (30\% vs $17,6 \% ; p=0,06)$.

Así mismo no obtuvimos diferencias en la mortalidad entre pacientes con TCE puro o asociado a politraumatismo, coincidiendo con el trabajo de Feickert et $\mathrm{al}^{25}$. Sin embargo, en el estudio de Tepas et $\mathrm{al}^{41}$, la mortalidad asociada al TCE con lesiones extracraneales casi duplicaba la del TCE puro.

Destacar como dato de relevancia también que la mortalidad en el TCEG pediátrico se produce generalmente de forma precoz, ya que su estancia en UMIP es significativamente menor que en los pacientes que no fallecen $(4,5$ días vs 10,$4 ; \mathrm{p}<0,001)$. Por ello consideramos que los factores que se deben analizar en relación con la mortalidad en los pacientes pediátricos deben ser factores precoces (al ingreso, primeras $24 \mathrm{~h}$ ), porque será este periodo de tiempo el que marcará el pronóstico en los niños con TCEG.

Por último en el apartado de la mortalidad del TCEG pediátrico, a pesar del problema que supone la homogeneización de muestras, en nuestro estudio existe una tendencia evidente a la disminución de la mortalidad en la última década $(25,5$ vs $15,1 \%)$, en la que pueden influir la optimización diagnóstico-terapéutica en la asistencia de los pacientes con TCEG, así como las mejoras en la seguridad vial, sistemas de inmovilización en los vehículos, etc.

En el análisis multivariable, la HIC o la existencia de midriasis uni o bilateral como reflejo de HIC y evento previo a la herniación cerebral fueron factores determinantes de mortalidad asociado a una alta odds ratio, hecho previamente descrito por otros autores ${ }^{5,7-9,32}$. A pesar de que estas 2 variables pueden plantear dudas como posibles variables de confusión (asociación entre ellas y asociación con la mortalidad), la regresión logística binaria (método stepwise) y el análisis de calibración (bondad de ajuste y estadístico $\chi^{2}$ de Hosmer-Lemeshow) realizados consideran a estas 2 variables factores independientes de mortalidad con un buen nivel de ajuste del modelo. Una tercera variable incluida en este análisis fue la hiperglucemia. El encéfalo en condiciones anaerobias produce un aumento de la producción de ácido láctico (glucólisis anaerobia) que favorece el aumento de la osmolaridad y edema cerebral ${ }^{36,37}$. En nuestro trabajo la hiperglucemia multiplica por 3 el riesgo de mortalidad en los niños con TCEG.

En conclusión, y a pesar del inconveniente de tratarse de un estudio en parte retrospectivo, podemos establecer la relevancia, en relación con la mortalidad, de la lesión 
neurológica grave (midriasis pupilar, HIC) y de factores favorecedores del edema cerebral como la hiperglucemia.

\section{Conflicto de intereses}

Los autores declaran no tener ningún conflicto de intereses.

\section{Bibliografía}

1. Adelson PD, Bratton SL, Carney NA, Chessnut RM, du Coudray $\mathrm{HE}$, Goldstein B, et al. Guidelines for the acute medical management of severe traumatic brain injury in infants, children and adolescents. Chapter 1: Introduction. Pediatr Crit Care Med. 2003;4:S2-4.

2. Keenan HT, Runyan DK, Marshall SW, Nocera MA, Merten D, Sinal S. A population-based study of inflicted traumatic brain injury in young children. JAMA. 2003;290:621-6.

3. Parslow RC, Morris KP, Tasker RC, Fossyth RJ, Hawley CA. Epidemiology of traumatic brain injury in children receiving intensive care in the UK. Arch Dis Child. 2005;90:1182-7.

4. Ponsford J, Willmott C, Rothwell A, Cameron P, Ayton G, Nelms $R$, et al. Impact of early intervention on outcome after mild traumatic brain injury in children. Pediatrics. 2001;108:1297-303.

5. Ono J, Yamaura A, Kubota M, Okimura Y, Isobe K. Outcome prediction in severe head injury:analices of clinical prognostic factors. J Clin Neurosci. 2001;8:120-3.

6. Keenan H, Nocera N, Braton S. Frequency of intracraneal pressure monitoring in infants and young toddlers with traumatic brain injury. Pediatr Crit Care Med. 2005;6:537-41.

7. Bahloul M, Chokri BH, Hedi C, Adel C, Harem K, Hassen D, et al. Severe head injury among children: prognostic factors and outcome. Injury. 2009;40:535-40.

8. Odeboe T, Abubakar A. Childhood head injury: causes, outcome and outcome predictors. Pediatr Surg Int. 2004;20:348-52.

9. White JR, Farukhi Z, Bull C, Christensen J, Gordon T, Paidas C, et al. Predictors of outcome in severely head-injured children. Crit Care Med. 2001;29:534-40.

10. Kochanek P, Clark R, Ruppel R, Adelson P, Bell M, Walen M, et al. Biochemical, cellular, and molecular mechanisms in the evolution of secondary damage after severe traumatic brain injury in infants and children: lesson learned from the beside. Pediatr Crit Care Med. 2000;1:4-19.

11. Jackson S, Piper I, Souter M. A study of the effects of using different cerebral perfusion pressure (CPP) thresholds to quantify CPP "secondary insults" in children. Acta Neurochir. 2000;76:453-6.

12. Teadsdale G, Jennet B. Assessment of coma and impaired consciousness. A practical scale. Lancet. 1974;2:81-4.

13. Reilly PL, Simpson DA, Sprod R, Thomas L. Assesing the conscious level of the Glasgow Coma Scale. Childìs Nerv Syst. 1988;4:30-3.

14. Pollack MM, Ruttimann UE, Getson PR. Pediatric risk of mortality (PRISM) score. Crit Care Med. 1988;16:1110-6.

15. Faist $F$, Baue $A E$, Dittmer $H$, Heberer $G$. Multiple organ failure in polytrauma patiens. J Trauma. 1983;23:775-87.

16. Henao F, Daes J, Dennis R. Risk factors for multiorgan failure. A case control study. J Trauma. 1991;31:74-80.

17. Foulkes MA, Eisemberg HM, Jane JA, and the Traumatic Coma Data Bank Group. The Traumatic Coma Data Bank: design, methods, and baseline characteristics. J Neurosurg. 1991;75:8-36.

18. López JM, Limiñana JM, Jiménez A, Consuegra E, Morón A, González R. Traumatismo craneoencefálico pediátrico grave. Factores predictores de mortalidad. Med Intensiva. 2003;27:155-61.
19. Cruz J. Combined continuos monitoring of systemic and cerebral oxignetion in acute brain injury: Preliminary observations. Crit Care Med. 1993;21:1225-32.

20. Jennett $B$, Bond $M$. Assessment of outcome after severe brain damage. Lancet. 1975;1:480-4.

21. Alted E, Bermejo S, Chico M. Actualizaciones en el manejo del traumatismo craneoencefálico grave. Med Intensiva. 2009;33:16-30.

22. Kochanek P, Tasker R. Pediatric neurointensive care: 2008 Update for the Rogers' Textbook of pediatric intensive care. Pediatr Crit Care Med. 2009;10:517-23.

23. Fernández A, Palomeque A, Cambra FJ, Ortega J, Costa JM, Caritg J, et al. Traumatismo craneoencefálico grave en el paciente pediátrico. Evaluación de la neuroimagen y monitorización de la presión intracraneal como factores pronóstico. Med Intensiva. 2001;25:8-13.

24. Reid SR, Roesler JS, Gaichas AM, Tsai AK. The epidemiology of pediatric traumatic brain injury in Minnesota. Arch Pediatr Adolesc Med. 2001;155:784-9.

25. Feickert HJ, Drommer S, Heyer R. Severe head injury in children: Impact of risk factors on outcome. J Trauma. 1999;47:33-8.

26. Esparza J, Portillo J, Sarabia M, Yuste J, Roger R, Lmas E. Outcome in children with severe head injuries. Childǐs Nerv Syst. 1985;1:109-14.

27. Chiaretti A, Piastra M, Pulitano S, Pietrini D, De Rosa G, Barbaro R, et al. Prognostic factors and outcome of children with severe head injury. An 8-year experience. Child's Nerv Syst. 2002;18:129-36.

28. Tilford JM, Simpson PM, Yeh TS, Lensing S, Aitken ME, Green $\mathrm{JW}$, et al. Variation in therapy and outcome for pediatrics head trauma patients. Crit Care Med. 2001;29:1056-61.

29. Salim A, Hannon M, Brown C, Hadjizacharias P, Backlus L, Texeira PG, et al. Intracranial pressure monitoring in isolated pediatric blunt head trauma. Am Surg. 2008;74: 1088-93.

30. Diamond IR, Parkin PC, Wales PW, Bohn D, Kreller MA, Dykes $\mathrm{EH}$, et al. Pediaric blunt and penetratin trauma deaths in Ontario: a population-based study. Ped J Pediatr Surg. 2009;44: 981-6.

31. Ward JD. Pediatric head injury: a futher experience. Pediatr Neurosurg. 1994;20:183-5.

32. Pillai S, Praharaj SS, Mohanty A, Kolluri VR. Prognostic factors in children with severe diffuse brain injuries: a study of 74 patients. Pediatr Neurosurg. 2001;34: 98-103.

33. Lang DA, Teasdale GM, Macpherson P, Lawrence A. Diffuse brain swelling after head injury: more often malignant in adults than children? J Neurosurg. 1994;80:675-80.

34. Marshall LF, Eisemberg HM, Jane JA, Luerssen TG, Marmarou A, Foulkes MA. The outcome of severe closed head injury. J Neurosurg. 1991;75:S28-36.

35. Manley G, Knudson MM, Morabito D, Damron S, Erikson V, Pitts L. Hypotension, hipoxia and head injury: frecuency, duration, and consequences. Arch Surg. 2001;136:1118-23.

36. Vespa P, Boonyaputthikul R, McArthur DL, Miler C, Etchepare $M$, Bergsneider $M$, et al. Intensive insuline therapy reduces microdyalisis glucose values withot altering glucose utilization or improving the lactate/pyruvate ratio after traumatic brain injury. Crit Care Med. 2006;34:850-6.

37. Rovlias A, Kotsou S. The influence of hyperglycemia on neurological outcome in patients with sever head injury. Neurosurg. 2000;46:335-43.

38. Mansfield RT. Head injuries in children and adults. Crit Care Clin. 1997;13:611-28.

39. Reviejo K, Arceaga I, Txoperena G, Azaldegui F, Alberdi F, Lara G. Análisis factores pronósticos de la mortalidad en el TCE grave. Proyecto Poliguitania Med Intensiva. 2002;26:241-7. 
40. Levi L, Guilbird JN, Linn S, Feinsod H. The association between skull fracture, intracranial pathology and outcome in pediatric head injury. $\mathrm{Br} \mathrm{J}$ Neurosurg. 1991;5: 617-25.

41. Tepas JJ, DiScala C, Ramenofsky ML, Barlow B. Mortality and head injury: The pediatric perspective. J Pediatr Surg. 1990;25:92-6.
42. Johnson DL, Krishnamurthy S. Severe pediatric head injury: Mith, magic, and actual fact. Pediatr Neurosurg. 1998;28: 167-72.

43. Kieslisch M, Marquardt G, Galow G, Lorenz R, Jacobit G. Neurological and mental outcome after severe head injury in childhood: a long term follow-up of 318 children. Disabil Rehabil. 2001;23:665-9. 